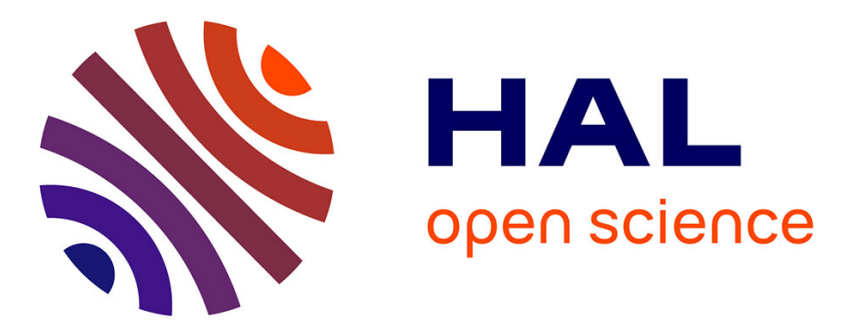

\title{
Fibrodysplasie ossifiante progressive et hétéroplasie ossifiante progressive
}

Geneviève Baujat, Caroline Michot, Kim-Hanh Le Quan Sang, Valérie

Cormier-Daire

\section{To cite this version:}

Geneviève Baujat, Caroline Michot, Kim-Hanh Le Quan Sang, Valérie Cormier-Daire. Fibrodysplasie ossifiante progressive et hétéroplasie ossifiante progressive. Revue du Rhumatisme monographies, 2019, 86, pp.40 - 45. 10.1016/j.monrhu.2018.11.003 . hal-03486559

\section{HAL Id: hal-03486559 \\ https://hal.science/hal-03486559}

Submitted on 20 Dec 2021

HAL is a multi-disciplinary open access archive for the deposit and dissemination of scientific research documents, whether they are published or not. The documents may come from teaching and research institutions in France or abroad, or from public or private research centers.
L'archive ouverte pluridisciplinaire HAL, est destinée au dépôt et à la diffusion de documents scientifiques de niveau recherche, publiés ou non, émanant des établissements d'enseignement et de recherche français ou étrangers, des laboratoires publics ou privés.

\section{다)(1) $(5$}

Distributed under a Creative Commons Attribution - NonCommerciall 4.0 International 


\title{
Fibrodysplasie Ossifiante Progressive et Hétéroplasie Ossifiante Progressive
}

\section{Fibrodysplasia Ossificans progressiva and Progressive Osseous Heteroplasia}

\author{
Geneviève Baujat, Caroline Michot, Kim-Hanh Le Quan Sang, Valérie Cormier-Daire
}

Service de Génétique Médicale et Institut Imagine, Centre de Référence Maladies Osseuses

Constitutionnelles, Hôpital Universitaire Necker-Enfants malades, 149 rue de Sèvres, 75015

Paris, France

Université Paris Descartes-Sorbonne Paris Cité,

Auteur principal

Geneviève Baujat : genevieve.baujat@aphp.fr

Téléphone : 0171196418 


\section{Résumé}

La Fibrodysplasie Ossifiante Progressive (FOP) ou maladie de Münchmeyer, est une pathologie génétique rare, autosomique dominante, se manifestant par des poussées d'ossification hétérotopique, progressives et cumulatives, aboutissant à une douloureuse métamorphose en «homme de pierre », ankylose irrémédiable laissant les familles et les professionnels de santé souvent désemparées devant l'immuable progression de la pathologie. L'implication d'une mutation récurrente du gène ACVR1/ALK2, mutation activatrice de la voie du (TGF)- $\beta$ / BMP a été démontré en 2006 par F. Kaplan, E. Shore et un consortium international. Cette découverte majeure a lancé de nombreux travaux de recherche permettant d'appréhender les bases physiopathologiques et d'aborder des pistes thérapeutiques spécifiques. Le suivi et la prise en charge de cette pathologie incluent pour l'instant essentiellement des mesures préventives et le traitement symptomatique des poussées inflammatoires douloureuses précédant le phénomène d'ossification. Plusieurs thérapeutiques innovantes sont en cours de développement préclinique et clinique, visant à bloquer les voies de signalisation d'amont et d'aval de ACVR1/ALK2. Elles marquent une nouvelle ère pour la communauté de personnes concernées engagées auprès des patients FOP.

L'hétéroplasie ossifiante progressive (Progressive Osseous Heteroplasia, POH, OMIM 166350) appartient aussi au groupe des ossifications hétérotopiques, caractérisé par la survenue anormale de formation osseuse au sein des tissus conjonctifs. Il s'agit d'une pathologie ultra-rare (moins de 100 cas décrits à ce jour), progressive, d'évolution très variable. Cette grande rareté semble être liée à une pénétrance incomplète avec large variabilité des manifestations. Le phénomène d'ossification est épisodique, comme dans la FOP. Par contre, ces deux pathologies sont distinguées par le fait que la POH implique un phénomène d'ossification principalement d'origine intramembraneuse, qu'il n'existe pas de facteur déclenchant aux poussées, qu'il n'existe pas la progression selon un schéma caractéristique prédictible et par l'absence de facteur inflammatoire concomitant. Il existe une différenciation directe des progéniteurs mésenchymateux en ostéoblastes. La POH est associée à des mutations inactivatrices du gène GNAS. Les traitements sont actuellement limités aux mesures symptomatiques, notamment assurer une asepsie cutanée, une antalgie adaptée et aux anti-inflammatoires non-stéroïdiens.

Mots clés: Fibrodysplasie Ossifiante Progressive, Hétéroplasie Ossifiante Progressive, ossification hétérotopique, hallux valgus, myosite ossifiante, BMP, ACVR1/ ALK2, GNAS 
Fibrodysplasia Ossificans Progressiva (FOP), or Münchmeyer's disease, is a rare, autosomal dominant genetic disorder, characterized by episodic progressive and cumulative heterotopic ossification, leading to a disabling metamorphosis into a "stone man", with irremediable joint immobility, leaving families and health professionals often distraught, faced to the immeasurable progression of the disease. The involvement of a recurrent mutation of the ACVR1/ALK2 gene, an activating mutation of the (TGF)- $\beta$ /BMP pathway, was demonstrated in 2006 by F. Kaplan, E. Shore and an international consortium. This major discovery has launched many research projects for understanding the pathophysiological basis and targeting specific therapies approaches. The present management of this pathology includes mainly preventive measures, and symptomatic treatment of disease flare-ups preceding the phenomenon of ossification, by various anti-inflammatory agents. Several innovative therapies are currently undergoing preclinical and clinical development, aimed at blocking the upstream and downstream signaling pathways of ACVR1 / ALK2, marking a new era for the community of affected individuals and people engaged with FOP patients.

Progressive Osseous Heteroplasia $(\mathrm{POH})$ also belongs to the group of genetic disorders with extensive heterotopic ossification, characterized by the abnormal formation of bone within the connective tissues. It is an ultra-rare pathology (less than 100 cases described to date), severely progressive. This great rarity seems to be related to incomplete penetrance with wide variability of manifestations. The onset of ossification is episodic, as in FOP. On the other hand, these two conditions may be distinguished by the facts that $\mathrm{POH}$ is due to heterotopic ossification mainly of intramembranous origin, that there is no triggering factor leading to bone formation, there is no progression according to a characteristic pattern and by the absence of concomitant inflammatory factor. There is a direct differentiation of mesenchymal progenitors into osteoblasts. $\mathrm{POH}$ is associated with inactivating mutations of the GNAS gene. Treatments are currently limited to symptomatic measures, including ensuring cutaneous asepsis, adequate analgesia and non-steroidal anti-inflammatory drugs.

Fibrodysplasia Ossificans Progressiva, Progressiva Ossificans Heteroplasia, heterotopic ossification, hallux valgus, myositis ossificans, BMP, ACVR1/ ALK2, GNAS 


\section{1/ Fibrodysplasie Ossifiante Progressive}

\subsection{Caractéristiques cliniques de la forme classique et histoire naturelle}

La FOP (OMIM 135100) est cliniquement caractérisée par l'association d'une malformation congénitale du gros orteil, court et déformé en valgus, à des épisodes de poussées inflammatoires entraînant le développement d'ossifications hétérotopiques dans les tissus conjonctifs et musculaires. Ces poussées surviennent à un âge variable, sont progressives et cumulatives, menant à une ankylose généralisée, des complications respiratoires et thromboemboliques et à un décès précoce à l'âge adulte [1].

Le diagnostic de FOP est exceptionnellement porté en anténatal [donnée non publiée]. Les nouveau-nés présentent des hallux valgus et parfois raccourcis [Fig. 1] [2], signe isolé et passant souvent inaperçu. Lors des premiers mois, peuvent survenir des nodules transitoires du scalp, tuméfactions parfois inflammatoires et douloureuses, disparaissant spontanément en quelques jours, sans laisser de signe dans la majorité des cas [3].

Les enfants présentent à partir d'un âge très variable, en général pendant la première décade, la succession de poussées inflammatoires («Flare-up»), au niveau des tissus conjonctifs, enthèses, muscles squelettiques et/ou leurs aponévroses et les capsules articulaires. Ce sont des tuméfactions douloureuses, accompagnées parfois des signes généraux (fièvre), progressant selon un schéma particulier (cranio-caudal, postéro-antérieur et proximo-distal) mimant le développement normal du squelette embryonnaire. Ces zones inflammatoires douloureuses laissent place dans la majorité des cas à des ankyloses localisées traduisant le phénomène d'ossification endochondrale hétérotopique. Elles concernent en général d'abord les régions cervicales et paravertébrales, puis les épaules, les régions dorsales puis lombaires [Fig. 2], les hanches, et enfin les membres. Les poussées peuvent être spontanées ou secondaires à un événement déclencheur, lésion musculaire ou pathologie virale de type grippale : vaccination intramusculaire, traumatisme ou hématome musculaire, chute, grippe. L'ablation chirurgicale d'une lésion inflammatoire peut entraîner une salve de poussées successives durant plusieurs mois. Les muscles lisses, le muscle cardiaque, et certains muscles squelettiques comme le diaphragme, les muscles périorbitaires et la langue sont épargnés [4$5]$.

D'autres événements cliniques marquent l'évolution de la FOP. Les enfants ont souvent une avance staturale avec avance d'âge osseux pendant les 10-15 premières années, jusqu'à ce que les ankyloses articulaires et musculaires limitent leur croissance. Une surdité (perception ou 
mixte) apparaît dans $50 \%$ des cas à l'âge pédiatrique tardif [5]. Des anomalies neurologiques et une augmentation de la fréquence des lithiases rénales ont été récemment décrits chez une faible minorité de patients $[6,7]$.

Pendant la seconde décade, les ossifications cumulatives, musculaires et périarticulaires entraînent des limitations progressives de la marche et des gestes de la vie quotidienne, avec perte progressive de l'indépendance. L'ankylose de la mâchoire et une fréquente rétrognathie progressive entraînent des difficultés alimentaires importantes. La plupart des patients sont confinés dans un fauteuil roulant lors de la troisième décade. Les complications de la quatrième décade comprennent les atteintes thromboemboliques (liées à l'immobilité et la stase veineuse) et cardiopulmonaires (conjugaison de la diminution des volumes pulmonaires, des possibles pneumopathies de reflux et des apnées du sommeil). Cet ensemble constitue le syndrome d'insuffisance thoracique pouvant aboutir au décès. L'âge médian de décès est actuellement d'environ 45 ans $[1,5]$.

Sur le plan radiologique, le signe le plus précoce est le monophalangisme du premier orteil, associés à un aspect trapu et élargi du premier métacarpe. La première phalange est en générale courte, triangulaire ou trapézoïde, parfois fusionnée avec le premier métatarsien ou avec la seconde phalange [Fig. 3]. Le premier rayon des mains est également en général raccourci, avec un premier métacarpien bref, et une hypoplasie des phalanges du pouce. La première phalange est souvent déformée, triangulaire, avec une ligne métaphysaire oblique en bas et en dehors. La seconde phalange peut être étroite, comme les autres phalanges terminales des autres doigts. Une brachymésophalangie des $\mathrm{V}$ est fréquente. Lors des premières années apparaissent des ostéochondromes (ou exostoses) fines et sessiles en région fémorale inférieure et tibiale supérieure. Un aspect allongé et étroit avec fusion des facettes articulaires des vertèbres $\mathrm{C} 2$ à $\mathrm{C} 7$ est fréquemment observé précocement, associé à une limitation de la mobilité cervicale, avant même l'apparition des premières poussées. Enfin, les cols fémoraux sont larges et trapus, contrastant avec une petite taille des épiphyses fémorales supérieures.

La grande variabilité de cette affection a été soulignée depuis longtemps. Il existe des formes d'expressivité précoce et d'autres se révélant à l'adolescence voir à l'âge adulte. Quel que soit l'âge d'apparition, il existe des formes lentement progressives et d'autres dont l'évolution est rapidement croissante. Les signes mineurs de FOP classique sont présents de façon variable (déficit auditif, ostéochondromes, syndrome douloureux chronique). Par ailleurs, il existe des formes de FOP atypiques associant des signes originaux comme des anomalies 
réductionnelles transverses, une alopécie, une atteinte ophtalmologique (cataracte, glaucome) ou encore des anomalies cérébrales avec possible déficience intellectuelle [8, 9].

\subsection{Epidémiologie et prévalence}

Il s'agit d'une affection ultra rare avec une prévalence rapportée de façon variable de 0,36 à 1.39 par million, sans facteur ethnique ou géographique prédisposant. [1, 10]. Les rares cas transmis obéissent à un mode d'hérédité autosomique dominante. Il existe une variabilité intrafamiliale liée à l'influence probable de facteurs environnementaux et les événements médicaux concomitants.

\subsection{Etiologie moléculaire}

La FOP est associée à une mutation mono-allélique gain de fonction de $A C V R 1$, gène codant pour un récepteur aux BMP (bone morphogenetic protein) 1A, également appelé activine-like kinase 2 (ALK2) et appartenant à la famille des TGF $\beta$. La mutation récurrente c.617G >A (p.Arg206His), au niveau du domaine Glycine-Sérine (GS) intracellulaire, est retrouvée chez la majorité des patients présentant une forme classique [11]. D'autres mutations de ce gène ont été décrites, associées à des formes de FOP atypiques [8]. Le récepteur mutant mACVR1 devient hypersensible aux ligands BMP, avec perte de l'auto-inhibition de la voie de signalisation BMP-Smad 1/5/8 et une réponse altérée à la modulation aux ligands des voies canoniques BMP et non canoniques Activine A. Des études récentes ont décrit en effet l'importance de cet autre ligand non canonique, Activine A, dans la survenue des ossifications hétérotopiques dans la FOP, ce ligand étant un inhibiteur antagoniste de la voie de signalisation BMP [12].

Il existe des arguments cliniques et physiopathologiques pour suggérer la place indéniable du système immunitaire dans la survenue des ossifications hétérotopiques. La présence de macrophages et de mastocytes dans les phases précoces des poussées, le lien entre les épisodes infectieux et la réponse aux anti-inflammatoires sont des éléments importants supportant la place du système immunitaire dans la pathogenèse de la FOP [13].

\subsection{Diagnostic, diagnostic différentiel}

Le diagnostic est fortement évoqué par les arguments clinico-radiologiques. Les clichés essentiels dépendent de l'âge et comportent en général les pieds, le bassin, le rachis cervical (profil) et les genoux. Il n'y a pas d'anomalie sur les examens biologiques de routine, notamment au niveau du bilan phosphocalcique et du bilan inflammatoire. La biopsie lésionnelle est fortement contre-indiquée. Lors des poussées, on peut trouver une augmentation transitoire des phosphatases alcalines en dehors d'un déficit en vitamine D. L'étude moléculaire par recherche de la mutation récurrente doit être réalisée lors d'une 
consultation par un généticien ou un expert de la pathologie. L'annonce diagnostique doit être particulièrement prudente et accompagnée auprès du patient et de sa famille. Si la mutation récurrente n'est pas retrouvée, le patient peut être référé en Centre de Référence Maladies Osseuses Constitutionnelles pour réévaluation diagnostique et éventuellement étude de la séquence entière de $A C V R 1$. Le pronostic de cette affection reste sombre en raison d'une lourde morbidité, de limitations fonctionnelles progressives et d'une mortalité pour l'instant précoce. [1, 5]

\subsection{Diagnostics différentiels}

Les principaux diagnostics différentiels sont les autres rares cas de myosites ossifiantes, notamment post traumatisme, la fibromatose juvénile, les sarcomes des tissus mous, les calcinoses tumorales et l'hétéroplasie osseuse progressive ( $\mathrm{POH})$.

\subsection{Prise en charge et suivi}

À ce jour il n'y a pas de traitement curatif définitif, et les traitements proposés lors des poussées n'ont pas fait preuve de parfaite efficacité. Néanmoins, le suivi régulier, les éléments de prévention des facteurs d'aggravation et le traitement symptomatique sont essentiels pour les personnes atteintes.

\subsubsection{Mesures préventives}

L'éviction des gestes invasifs, des chutes et traumatismes, des infections notamment grippales constituent des mesures majeures. Les chirurgies requises pour les situations d'urgences font appels à des anesthésistes expérimentés (avec intubation nasotrachéale sous contrôle scopique), et à l'adjonction de corticoïdes en pré et postopératoire. Les chutes sont fréquentes, souvent dès la première décade, favorisées par le trouble de l'équilibre sagittal lié aux déformations, et aggravées par l'absence de retenue par les bras ankylosés. Chaussures légères antidérapantes (et parfois avec semelles de compensation), adaptations du domicile avec éviction des obstacles et kinésithérapie de proprioception sont des éléments importants au quotidien. Les vaccinations intramusculaires sont en général proscrites à partir du moment où le diagnostic est posé. Chez le sujet plus âgé, fragile, on peut discuter la vaccination antigrippale par voie sous-cutanée superficielle dans une zone non mobile [1].

Les soins réguliers de la cavité buccale et des dents sont essentiels pour prévenir des complications infectieuses. Les anesthésies peuvent être réalisées sur les dents de lait par infiltration à travers la pulpe dentaire, sur les dents définitives par micro-injections interligamentaires réalisées de façon très prudente. Dans certains cas, les soins dentaires sont plus facilement réalisés sous anesthésie générale avec intubation nasotrachéale vigile en position semi assise, après anesthésie locale (spray ou méchage) [14]. 


\subsubsection{Traitement d'une poussée}

Les corticoïdes sont actuellement proposés pour réduire l'inflammation des poussées survenant près des grosses articulations, au niveau de la mâchoire et de la région sous maxillaire. Ils doivent être donnés le plus précocement possible, à forte dose pendant 4 jours (par exemple Prednisone $2 \mathrm{mg} / \mathrm{kg}$ / jour sans dépasser $100 \mathrm{mg}$, en une prise matinale) suivie d'une décroissance progressive sur une durée de 10-15 jours. On peut également proposer un traitement initial par méthylprednisolone par voie intraveineuse, $2 \mathrm{mg} / \mathrm{kg} / \mathrm{jour}$ en intraveineux direct, pendant 3 jours, puis relais per os. Ceci nécessite une entente préalable avec le service d'urgence de l'hôpital de proximité. Les cures de corticoïdes ne sont pas indiquées pour les poussées des régions dorsale et lombaire, cervicale postérieure, et du thorax, par leur nature souvent récurrente et la difficulté à les identifier.

Les antiinflammatoires non-stéroïdiens peuvent être prescrits en cours de diminution du traitement par corticoïdes, afin de poursuivre l'effet inflammatoire, pendant plusieurs semaines selon le contexte. Les inhibiteurs sélectifs de la COX-2 sont particulièrement indiqués chez les patients adultes ne présentant pas d'insuffisance cardiaque. D'autres classes thérapeutiques comme les bisphosphonates, les inhibiteurs des mastocytes et les inhibiteurs de protéine tyrosine kinase (Imatinib, Glivec $®$ ) ont pu être essayés de façon compassionnelle, sur de petites séries, avec des résultats encore limités [15].

\subsubsection{Essais thérapeutiques en cours}

Grâce aux avancées des connaissances de la physiopathologie de la FOP et la mise au point de modèles murins adéquats, de nouvelles molécules sont également aujourd'hui en phase de développement préclinique et clinique. Ces nouvelles stratégies sont orientées vers le blocage de l'activité du récepteur muté ou de ses ligands d'amont, l'interférence avec les facteurs déclencheurs inflammatoires, ou encore le blocage d'aval de l'ossification BMP-dépendante [16]. En France, plusieurs sites du Centre de Référence Maladies Osseuses Constitutionnelles sont ou seront bientôt impliqués dans deux essais, d'une part le Palovarotène, agoniste du récepteur à l'acide rétinoïque de type gamma et puissant inhibiteur de l'ossification endochondrale (ClinicalTrials.gov Identifier: NCT02521792), et d'autre part, l'anticorps antiactivine A, REGN2477, visant à diminuer ce ligand en amont du récepteur muté (ClinicalTrials.gov Identifier: NCT03188666). On souligne également l'apparition récente d'études cliniques d'histoire naturelle, grâce à des registres de patients, permettant une meilleure connaissance des facteurs de variabilité de cette pathologie hétérogène, et la mise en place de guides de suivis adaptés à la pathologie [17].

\subsubsection{Les mesures symptomatiques}


Elles prennent une part importante chez les patients, notamment chez les plus âgés, avec notamment la kinésithérapie régulière articulaire et respiratoire, les méthodes d'antalgie médicamenteuse et non médicamenteuse, l'ergothérapie, les soins cutanés, la prise en charge cardiorespiratoire avec possibilité de ventilation non invasive nocturne, l'appareillage auditif, ou encore le traitement des complications thromboemboliques.

\subsection{5 Éléments de suivi, accompagnement scolaire et familial}

Le suivi médical est adapté à l'âge. Sur le plan fonctionnel, le score CAJIS (Cumulative Analogue Joint Involvement Scale) est intéressant pour suivre l'évolution des ankyloses articulaires [18]. Les radiographies standards localisées sont peu utiles pour le suivi des ossifications ectopiques. Le scanner est un bon outil d'évaluation mais ne peut être proposé en routine à cause de l'irradiation induite. Les radiographies en technique EOS sont probablement à évaluer dans le futur. Il est important de surveiller régulièrement les fonctions respiratoires (EFR, PSG), cardiaques (ECG et échographie cardiaques) et auditives. Il n'existe pas de marqueurs biologiques de surveillance. Les mesures d'accompagnement socioéducatives et psychologiques sont primordiales pour permettre d'optimiser l'inclusion scolaire puis professionnelle, et/ ou la possibilité d'activités occupationnelles, les déplacements par des fauteuils personnalisés, et les adaptations nécessaires régulièrement revus au domicile [19].

Enfin, il est important d'informer les patients et leurs familles des groupes de patients en France (www.fopfrance.fr), en Europe (au sein de Eurordis, www.eurordis.org) et dans le monde (www.ifopa.org), véritable maillage très fort permettant à toute la communauté FOP de progresser ensemble vers l'amélioration des connaissances et des traitements de cette pathologie.

\section{2/ Hétéroplasie Osseuse Progressive}

\subsection{Bases physiopathologiques}

Le gène GNAS est situé en 20q13.32, au niveau d'un locus complexe, soumis à empreinte, comprenant de nombreux promoteurs, et associé à de nombreuses pathologies selon la régulation transcriptionelle impliquée [20]. Ce locus code pour cinq transcrits alternatifs dont l'expression est régulée de manière parentale, tissulaire et développementale : la sous-unité alpha stimulatrice de la protéine G (Gs $\alpha)$, XLas, NESP55, et deux ARNnc, A/B et GNASAS1. Gs $\alpha$ est une protéine ubiquitaire clé dans la transduction hormonale, partageant avec XLas la capacité de produire l'AMP cyclique intracellulaire après stimulation des récepteurs couplés à Gs $\alpha$, via l'activation d'adényl cyclases. Il existe une transcription préférentielle de 
l'allèle maternel de Gs $\alpha$ dans certaines cellules et tissus. Les mutations au locus GNAS sont associées à la pseudohypoparathyroidie 1A (PHP) et la pseudo-PHP. Les défauts de méthylation (épimutations) de GNAS causent la PHP1B autosomique dominante et sporadique. La PHP1a est associée à des mutations inactivatrices de l'allèle maternel de GNAS [21, 22]. La POH est associée à des mutations inactivatrices hétérozygotes de GNAS, comme l'ostéoma cutis (OC) et l'ostéoarthropathie héréditaire d'Albright (Albright Hereditary Osteodystrophy, AHO), mais, à la différence de ces deux entités alléliques, il s'agit presque exclusivement de mutations de l'allèle paternel de GNAS et l'ossification ectopique ne se limite pas aux tissus sous-cutanés et au derme [23, 24].

\subsection{Clinique}

Il n'y a pas de signe distinctif congénital. Les phénomènes d'ossification sont spontanés, et surviennent à un âge variable, sans facteur déclenchant identifié. Les premières lésions sont souvent discrètes, telles des papules érythémateuses proches de celles rencontrées dans l'OC et l'AHO. La coalescence de ces lésions, plus ou moins rapidement, va former des plaques extensives traduisant le phénomène d'ossification sous-cutanée. Il existe une progression de l'ossification du derme superficiel vers les tissus conjonctifs profonds et notamment dans les muscles. Il peut y avoir une fusion avec le squelette sous-jacent. La POH n'est pas associée à un phénomène inflammatoire. En histologie, les dépôts osseux sont en amas irréguliers au sein du derme, souvent à proximité du tissu adipeux sous-cutané. Il n'y a pas de signe clinique, biologique ou histologique d'inflammation. La sévérité est très variable, selon l'importance des lésions et leur localisation. Dans les formes majeures, l'atteinte des compartiments profonds peut entraîner des déformations, notamment une scoliose et des incurvations des membres, et un déficit de croissance [25]. Dans ces formes, les articulations s'ankylosent et il peut apparaître des ulcérations cutanées. L'évolution est variable, avec possibles phases de stabilisation après la puberté. Il existe une insuffisance respiratoire séquellaire combinée chez les sujets ayant une rigidité marquée du tronc avec déformations, pouvant mettre en jeu le pronostic vital. Il n'existe pas d'atteinte intellectuelle dans la $\mathrm{POH}$ [26].

La progression des lésions de POH est épisodique sur plusieurs années, selon un schéma anatomique particulier, en mosaïque, de distribution souvent hémimélique sur le tronc ou les membres. Il peut y avoir une atteinte bilatérale [27]. Ces lésions sont typiquement des amas d'ossification intramembraneux, mais des ilots de cartilage ectopique ont été démontrés [24]. 
Sur le plan radiologique, le réseau d'ossifications sous-cutané puis musculaire est parfois impressionnant, véritable maillage irrégulier extensif emprisonnant le squelette. On retrouve fréquemment une ostéoporose dans les formes majeures.

Les formes familiales sont exceptionnelles mais deux cas avec transmission dominante documentés ont été décrits, avec large variabilité intrafamiliale, et présence dans une même famille de forme a minima avec maculo-papules ossifiées de petite taille et de formes majeures sévères, suggérant soit la variabilité d'expressivité génétique et/ou phénomène de mosaïcisme chez un des parents présentant une forme peu sévère [28].

Il n'existe pas de résistance hormonale ni d'obésité dans la $\mathrm{POH}$.

\subsection{Diagnostics différentiels}

Les principaux diagnostics différentiels sont la FOP, l'AHO et les maladies de système (lupus, dermatomyosite) avec calcinosis universalis, pathologie dont la physiopathologie reste inconnue à ce jour, mais dont l'aspect radiologique et la présence d'un syndrome inflammatoire permettent souvent d'en faire la différence [26].

\subsection{Prise en charge, traitement}

Le traitement de la $\mathrm{POH}$ est toujours décevant à ce jour et il n'existe pas de thérapie spécifique. Il apparaît difficile de développer des agents compensant l'activité insuffisante de GNAS, d'autant plus du mécanisme d'empreinte parentale. Les principaux éléments de prise en charge sont d'assurer une bonne asepsie cutanée, de préserver les mobilités articulaires dans les phases d'exacerbation par une kinésithérapie douce et active, et tous les soins de support symptomatiques / prévention des complications de décubitus chez une personne possiblement douloureuse et dont la mobilité est réduite. Les manipulations forcées des articulations peuvent entraîner des microtraumatismes et hémorragies, facteurs de risque d'ossification hétérotopique. L'ablation des ossifications et les greffes cutanées n'apportent pas de bénéfice. Les anti-inflammatoires non stéroïdiens, notamment l'indométacine et les inhibiteurs COX-2 pourraient avoir un rôle dans la prévention des ossifications. Les bisphosphonates n'ont pas d'effet démontré sur les ossifications déjà constituées [26, 28].

Le conseil génétique pour les parents et la descendance de la personne atteinte est très difficile à cause de la régulation différentielle liée à l'empreinte parentale de la région de $G N A S$, et à l'hétérogénéité phénotypique associée à une même mutation de GNAS. Il existe probablement d'autres facteurs comme le patrimoine génétique, des événements épigénétiques et/ou des facteurs environnementaux contribuant à moduler la sévérité des ossifications hétérotopiques. Typiquement, le tableau de $\mathrm{POH}$ est potentiellement conséquent d'une mutation de l'allèle paternel de GNAS, mutation de novo ou transmise par un père porteur asymptomatique, alors 
que les mutations de l'allèle maternel entraînent essentiellement un tableau d'AHO. De nombreuses inconnues persistent pour expliquer la grande proportion de porteurs de mutation de l'allèle paternel de GNAS paucisymptomatiques, les $\mathrm{POH}$ associée à une mutation de l'allèle maternel de $G N A S$, et la distribution des lésions selon une distribution suivant le dermatomyome du somite avec latéralisation préférentielle [21]. L'hypothèse d'une pathologie avec mosaïcisme segmentaire de type 2 a été suggérée par Happle, phénomène génétique dans lequel il y a perte d'hétérozygotie acquise pendant les phases précoces de développement embryonnaire [21, 29]. Plusieurs études sont nécessaires pour poursuivre l'élucidation des mécanismes d'ossification ectopique sous-jacents dans les pathologies associées aux défauts de GNAS et les thérapeutiques potentiellement spécifiques.

Mention des conflits d'intérêt : Les auteurs ne déclarent aucun conflit d'intérêt en lien avec l'article 


\section{Références}

1. Kaplan FS, Le Merrer M, Glaser DL, et al. Fibrodysplasia ossificans progressiva. Best Pract Res Clin Rheumatol. 2008;22:191-205.

2. Kaplan FS, Xu M, Glaser DL, et al. Early diagnosis of fibrodysplasia ossificans progressiva. Pediatrics. 2008;121:1295-300.

3. Piram M, Le Merrer M, Bughin V, et al. Scalp nodules as a presenting sign of fibrodysplasia ossificans progressiva: a register-based study. J Am Acad Dermatol. 2011;64:97-101.

4. Pignolo RJ, Shore EM, Kaplan FS. Fibrodysplasia ossificans progressiva: clinical and genetic aspects. Orphanet J Rare Dis. 2011;6:80.

5. Pignolo RJ, Bedford-Gay C, Liljesthröm M, et al. The Natural History of Flare-Ups in Fibrodysplasia Ossificans progressiva (FOP): A Comprehensive Global Assessment. J Bone Miner Res. 2016;31:650-6.

6. Kitterman JA, Strober JB, Kan L, et al. Neurological symptoms in individuals with fibrodysplasia ossificans progressiva. J Neurol. 2012;259:2636-43.

7. Gupta RR, Delai PLR, Glaser DL, et al. Prevalence and risk factors for kidney stones in fibrodysplasia Ossificans Progressiva. Bone. 2018;109:120-123.

8. Kaplan FS, Xu M, Seemann P, et al. Classic and atypical fibrodysplasia ossificans progressiva (FOP) phenotypes are caused by mutations in the bone morphogenetic protein (BMP) type I receptor ACVR1. Hum Mutat. 2009;30:379-90.

9. Kaplan FS, Kobori JA, Orellana C, et al. Multi-system involvement in a severe variant of fibrodysplasia ossificans progressiva (ACVR1 c.772G >A; R258G): A report of two patients. Am J Med Genet A. 2015;167A:2265-71.

10. Baujat G, Choquet R, Bouée S, et al. Prevalence of fibrodysplasia ossificans progressiva (FOP) in France: an estimate based on a record linkage of two national databases. Orphanet J Rare Dis. 2017;12:123.

11. Shore EM, Xu M, Feldman GJ, et al. A recurrent mutation in the BMP type I receptor ACVR1 causes inherited and sporadic fibrodysplasia Ossificans progressiva. Nat Genet. 2006;38:525-7.

12. Hatsell SJ, Idone V, Wolken et al. ACVR1R206H receptor mutation causes fibrodysplasia ossificans progressiva by imparting responsiveness to activin A. Sci Transl Med. 2015;7:303ra137. 
13. Del Zotto G, Antonini F, Azzari I, et al. Peripheral Blood Mononuclear Cell Immunophenotyping in Fibrodysplasia Ossificans Progressiva Patients: Evidence for Monocyte DNAM1 Up-regulation. Cytometry B Clin Cytom. 2018;94:613-622.

14. Kilmartin E, Grunwald Z, Kaplan FS, et al. General anesthesia for dental procedures in patients with fibrodysplasia ossificans progressiva: a review of 42 cases in 30 patients. Anesth Analg. 2014;118:298-301.

15. Kaplan FS, Andolina JR, Adamson PC, et al. Early clinical observations on the use of imatinib mesylate in FOP: A report of seven cases. Bone. 2018;109:276-280.

16. Pignolo RJ, Shore EM, Kaplan FS. Fibrodysplasia ossificans progressiva: diagnosis, management, and therapeutic horizons. Pediatr Endocrinol Rev. 2013;10 Suppl 2:43748.

17. Mantick N, Bachman E, Baujat G, et al. The FOP Connection Registry: Design of an international patient-sponsored registry for Fibrodysplasia Ossificans Progressiva. Bone. 2018;109:285-290.

18. Kaplan FS, Al Mukaddam M, Pignolo RJ. A cumulative analogue joint involvement scale (CAJIS) for fibrodysplasia ossificans progressiva (FOP). Bone. 2017;101:123128.

19. Di Rocco M, Baujat G, Bertamino M, et al. International physician survey on management of FOP: a modified Delphi study. Orphanet J Rare Dis. 2017;12:110.

20. Kaplan FS, Shore EM. Progressive osseous heteroplasia. J Bone Miner Res. 2000 ;15:2084-94.

21. Bastepe M. GNAS mutations and heterotopic ossification. Bone. 2018;109:80-85.

22. Eddy MC, Jan De Beur SM, et al. Deficiency of the alpha-subunit of the stimulatory G protein and severe extraskeletal ossification. J Bone Miner Res. 2000;15:2074-83.

23. Shore EM, Ahn J, Jan de Beur S, et al. Paternally inherited inactivating mutations of the GNAS1 gene in progressive osseous heteroplasia. N Engl J Med. 2002;346:99106.

24. Adegbite NS, Xu M, Kaplan FS, et al. Diagnostic and mutational spectrum of progressive osseous heteroplasia $(\mathrm{POH})$ and other forms of GNAS-based heterotopic ossification. Am J Med Genet A. 2008;146A:1788-96.

25. Demir MK, Toktaş ZO, Akakın A, et al. Progressive osseous heteroplasia and scoliosis. Spine J. 2015;15:19-22.

26. Pignolo RJ, Ramaswamy G, Fong JT, et al. Progressive osseous heteroplasia: diagnosis, treatment, and prognosis. Appl Clin Genet. 2015;8:37-48. 
27. Urtizberea JA, Testart H, Cartault F, et al. Progressive osseous heteroplasia. Report of a family. J Bone Joint Surg Br. 1998;80:768-71.

28. Mantovani G, Bastepe M, Monk D, et al. Diagnosis and management of pseudohypoparathyroidism and related disorders: first international Consensus Statement. Nat Rev Endocrinol. 2018;14:476-500.

29. Happle R. Progressive osseous heteroplasia is not a Mendelian trait but a type 2 segmental manifestation of GNAS inactivation disorders: A hypothesis. Eur J Med Genet. 2016;59:290-4. 


\section{Figure 1}

Pied caractéristique avec hallux valgus chez une petite fille de 4 ans ayant une FOP.

\section{Figure 2}

Lésions d'ossification ectopique faisant saillie dans le dos d'un jeune patient de 5 ans ayant une FOP récemment diagnostiquée.

\section{Figure 3}

Cliché radiologique du pied droit d'un patient de 7 ans, ayant une FOP, avec un aspect trapu du premier métatarse, et une forme triangulaire et raccourcie de la première phalange, entraînant la déviation en valgus du premier rayon du pied. 




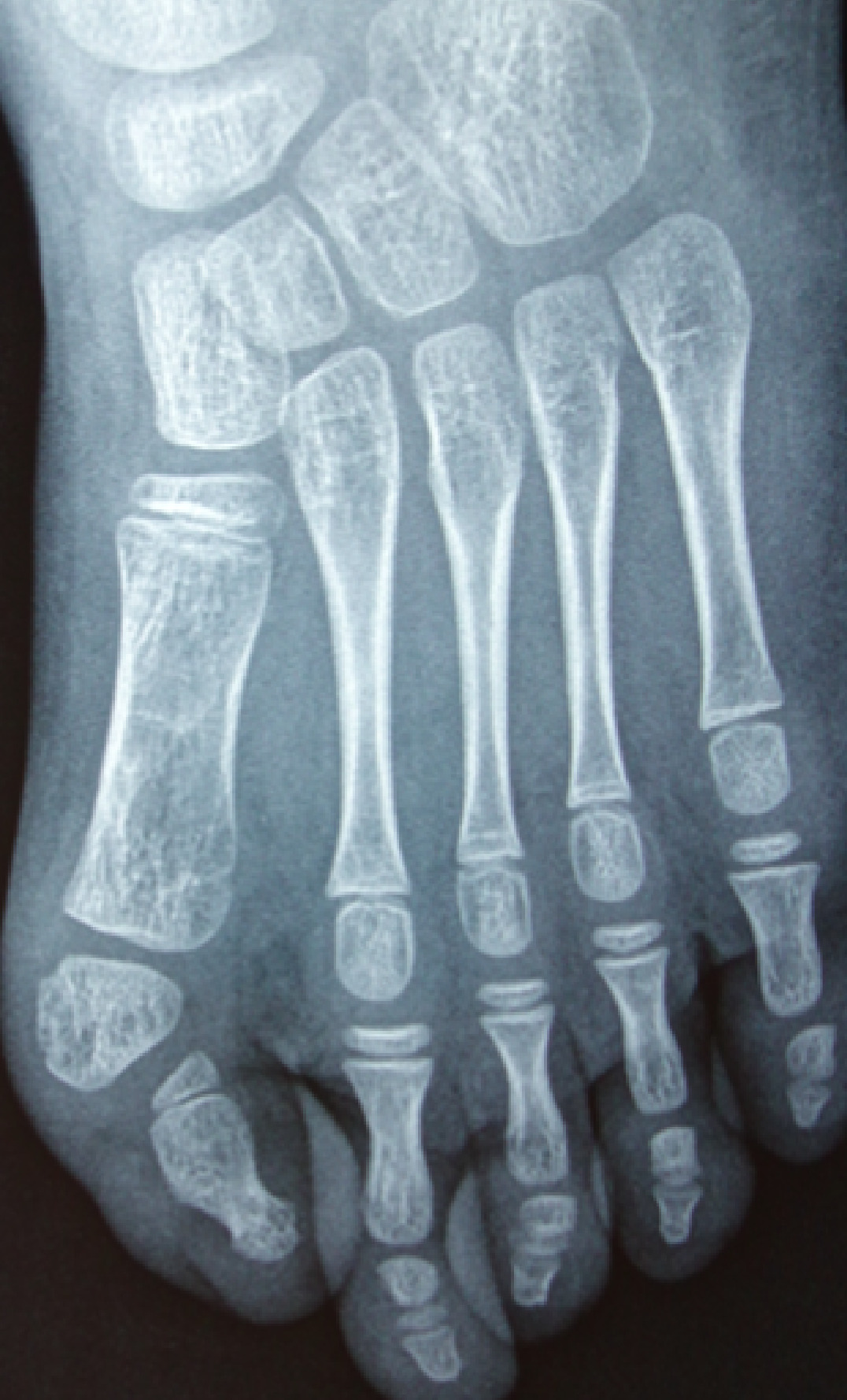

